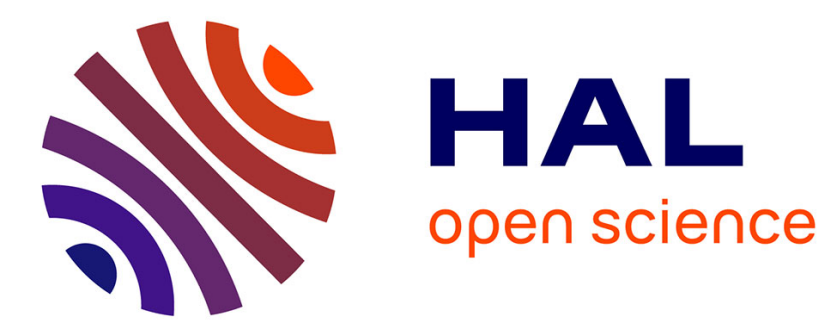

\title{
Event triggering strategies for consensus in clustered networks
}

Jihene Ben Rejeb, Irinel-Constantin Morarescu, Jamal Daafouz

\section{To cite this version:}

Jihene Ben Rejeb, Irinel-Constantin Morarescu, Jamal Daafouz. Event triggering strategies for consensus in clustered networks. 14th European Control Conference, ECC'15, Jul 2015, Linz, Austria. hal-01141220

\author{
HAL Id: hal-01141220 \\ https://hal.science/hal-01141220
}

Submitted on 12 Apr 2015

HAL is a multi-disciplinary open access archive for the deposit and dissemination of scientific research documents, whether they are published or not. The documents may come from teaching and research institutions in France or abroad, or from public or private research centers.
L'archive ouverte pluridisciplinaire HAL, est destinée au dépôt et à la diffusion de documents scientifiques de niveau recherche, publiés ou non, émanant des établissements d'enseignement et de recherche français ou étrangers, des laboratoires publics ou privés. 


\title{
Event triggering strategies for consensus in clustered networks
}

\author{
Jihene Ben Rejeb, Irinel-Constantin Morărescu and Jamal Daafouz
}

\begin{abstract}
This paper focuses on consensus in networks partitioned in several clusters. It uses the multi-agent framework in which the network is seen as a sum of interconnected subsystems called agents. We assume that each agent updates its state continuously by taking into account the states of some other agents belonging to the same cluster. This protocol allows reaching only local agreements in the network. In order to get consensus we endow an agent per cluster with the capacity to discretely interact outside its own cluster. The discrete interaction of one agent with agents from other clusters is modeled as a state jump or reset. The inter-clusters interactions are activated by using event dependent rules. The goal of the paper is to design event triggering reset strategies that guarantee the consensus is achieved. No global dwell time separating the reset instants is imposed but we show that a dwell time per cluster is ensured by the proposed reset strategies.
\end{abstract}

\section{INTRODUCTION}

Analysis and control of multi-agent systems attracted a lot of researchers during the last decade. This is certainly due to the omnipresence and variety of networks in our daily life. The multi-agent formalism allows treating applications coming from a wide spectrum of domains such as biology [1], [2], sociology [3], [4], [5], economy [6] or engineering [7], [8]. Each agent has only a local, limited information on the overall network, which means it applies decentralized strategy to reach a global goal.

The most studied problem on this topic is synchronization or consensus. It was mainly studied for linear agents interacting through a directed or an undirected graph with a fixed or dynamically changing topology [9], [10], [11]. Nevertheless, the literature on nonlinear agents is also very reach considering coupled oscillators dynamics [12], [13], [14], nonholonomic robots [15], [8] but also general dynamics encompassing the previous ones [16]. A basic assumption in most of the multi-agent systems studies is the network connectivity. There also exist works designing the controllers that are able to preserve the network connectivity [17] or topology [18]. Networks that asymptotically can loose connectivity were also analyzed [5], [19].

In this paper we consider that networks are partitioned in several clusters that are almost all the time isolated one from another. Each cluster is represented by a graph that is connected in a weak manner that will be defined in the next section. The agents continuously update their state by taking into account the states of other agents belonging to the

This work was funded by the ANR project COMPACS - "Computation Aware Control Systems", ANR-13-BS03-004.

The authors are with Université de Lorraine, CRAN, UMR 7039, 2 Avenue de la Forêt de Haye, France and CNRS, CRAN, UMR 7039. \{jihene.benrejeb, constantin.morarescu, jamal.daafouz\} at univ-lorraine.fr same cluster. This protocol allows reaching local agreements different from one cluster to another. To enforce consensus we assume that each cluster contains an agent endowed with better interaction capabilities that enable it to exchange information outside its cluster.

As explained in [20], this model can be interpreted in terms of opinion dynamics. Precisely, many social networks are structured in clusters and decision making process can be simplified as rapid/continuous-time iterations inside clusters accompanied by rare/discrete-time interactions between leaders of the clusters. The model can also be used in consensus for networks of robots spatially partitioned in clusters. Due to sensing limitation and energy saving the agents interact continuously only locally - inside clusters. From time to time, discretely, long distance interactions are afforded by a small number of agents that reset their state by taking into account the information received from other agents outside their cluster.

It is worth noting that in [20] all the resets were synchronized and the reset sequence was time dependent. The main assumptions were the time-separation and persistence of resets. Thus, the periods between consecutive resets of the overall multi-agent system were uniformly bounded. In [21] the authors also considered time-dependent resets but the reset sequences are independently defined for each cluster. This leads to asynchronous resets satisfying a dwell-time condition only inside each cluster but a global dwell-time lower bounding the period between consecutive resets of the overall multi-agent system no longer exist.

Unlike [20], [21], in this paper we design event based reset sequences. Event and self triggering strategies already exists in the literature [22], [23]. The main difference here is that replacing the discrete event triggering dynamics by continuous dynamics would lead in our case to system instability. The events triggering the resets are independently defined for each cluster leading to asynchronous reset sequences. The event-triggering strategy is mainly motivated by the fact that it avoids unnecessary and costly inter-clusters interactions.

The remaining paper is organized as follows. In Section II we introduce the concepts and we mathematically formalize the problem. Section III introduces the assumptions of work and recall some existing results that will be used in the sequel. The main contributions of the paper which is the design and analysis of reset strategies guaranteeing consensus are presented in Section IV. Numerical illustration are provided in Section $\mathrm{V}$ before concluding the paper.

Notation. The following standard notation will be used throughout the paper. The set of nonnegative integers, real and nonnegative real numbers are denoted by $\mathbb{N}, \mathbb{R}$ and $\mathbb{R}_{+}$, 
respectively. For a vector $x$ we denote by $\|x\|$ its Euclidian norm. The transpose of a matrix $A$ is denoted by $A^{\top}$. Given a symmetric matrix $A \in \mathbb{R}^{n \times n}$, notation $A>0(A \geq 0)$ means that $A$ is positive (semi-)definite. By $I_{k}$ we denote the $k \times k$ identity matrix. $\mathbf{1}_{k}$ and $\mathbf{0}_{k}$ are the column vectors of size $k$ having all the components equal 1 and 0 , respectively. We also use $x\left(t_{k}^{-}\right)=\lim _{t \rightarrow t_{k}, t \leq t_{k}} x(t)$.

\section{PROBLEM FORMULATION}

\section{A. Graph theory prerequisites}

We consider a network of $n$ agents described by the digraph (i.e. directed graph) $\mathcal{G}=(\mathcal{V}, \mathcal{E})$ where the vertex set $\mathcal{V}$ represents the set of agents and the edge set $\mathcal{E} \subset \mathcal{V} \times \mathcal{V}$ represents the interactions.

Definition 1: A directed path of length $p$ in a given digraph $\mathcal{G}=(\mathcal{V}, \mathcal{E})$ is a union of directed edges $\bigcup_{k=1}^{p}\left(i_{k}, j_{k}\right)$ such that $i_{k+1}=j_{k}, \forall k \in\{1, \ldots, p-1\}$. The node $j$ is connected with node $i$ in a digraph $\mathcal{G}=(\mathcal{V}, \mathcal{E})$ if there exists at least a directed path in $\mathcal{G}$ from $i$ to $j$ (i.e. $i_{1}=i$ and $j_{p}=j$ ). A strongly connected digraph is such that any of its two distinct elements are connected. A strongly connected component of a digraph is a maximal subset of $\mathcal{V}$ such that any of its two distinct nodes are connected. We say node $i$ is a parent of node $j$ in the digraph $\mathcal{G}=(\mathcal{V}, \mathcal{E})$ if $(i, j) \in \mathcal{E}$. A directed tree is a directed subgraph in which there exists a single node without parents called root while all the others have exactly one parent. A directed spanning tree of a digraph is a directed tree that links all the nodes of the graph. A digraph $\mathcal{G}$ is called weakly connected if it contains at least one spanning tree. For a given graph $\mathcal{G}=(\mathcal{V}, \mathcal{E})$, the subgraph induced by a subset of nodes $\mathcal{U} \subseteq \mathcal{V}$ is the graph $(\mathcal{U}, \mathcal{E} \cap(\mathcal{U} \times \mathcal{U}))$

In the sequel, we consider that the vertex set $\mathcal{V}$ is partitioned in $m$ clusters $\mathcal{C}_{1}, \ldots, \mathcal{C}_{m}$. We denote by $n_{i}$ the cardinality of each cluster $\mathcal{C}_{i}$.

Let us also introduce the intra-cluster graph $\mathcal{G}_{L}=\left(\mathcal{V}, \mathcal{E}_{L}\right)$ containing only the edges of $\mathcal{G}$ that connect agents belonging to the same cluster. That is

$$
\mathcal{E}_{L}=\left\{(i, j) \in \mathcal{E} \mid \exists k \in\{1, \ldots, m\} \text { such that } i, j \in \mathcal{C}_{k}\right\} .
$$

\section{B. System dynamics}

The state of each agent evolves continuously by taking into account the states of other agents belonging to their cluster. Doing so, the agents approach local agreements which can be different from one cluster to another. In order to reach the consensus in the entire network every inter-cluster connection is activated at some discrete instants. When the inter-cluster link $(j, i) \in \mathcal{E} \backslash \mathcal{E}_{L}$ is activated, the state of agent $i$ is reset to a weighted average of the states of $i$ and $j$. If several links arriving at $i$ are activated simultaneously, all the source states of these edges are considered in the weighted average. In order to keep the presentation simple each agent will have a scalar state denoted by $x_{i}$. We also introduce the vectors $x=\left(x_{1}, \ldots, x_{n}\right)^{\top} \in \mathbb{R}^{n}$ collecting the states of all the agents and $x_{\mathcal{C}_{i}}$ collecting the states of the agents belonging to cluster $i$, respectively.
Let $\mathcal{T}$ be the countable set of reset instants which are described by the diverging and increasing sequence $\left(t_{k}\right)_{k}$. Thus, the previous discussion is formally described by the linear reset system defining the overall network dynamics:

$$
\begin{cases}\dot{x}(t)=-L x(t), & \forall t \in \mathbb{R}_{+} \backslash \mathcal{T} \\ x\left(t_{k}\right)=P\left(t_{k}\right) x\left(t_{k}^{-}\right) & \forall k \in \mathbb{N} \\ x(0)=x_{0} & \end{cases}
$$

where $x_{0} \in \mathbb{R}^{n}, L \in \mathbb{R}^{n \times n}$ is a generalized Laplacian matrix associated to the intra-cluster graph $\mathcal{G}_{L}$ and $P\left(t_{k}\right) \in \mathbb{R}^{n \times n}$ is a stochastic matrix associated to the inter-cluster graph $\mathcal{G}_{P}\left(t_{k}\right)=\left(\mathcal{V}, \mathcal{E}_{P}\left(t_{k}\right)\right)$ where $\mathcal{E}_{P}\left(t_{k}\right) \neq \emptyset$ is the set of intercluster links activated at time $t_{k}$, so that $\mathcal{E}_{P}\left(t_{k}\right) \subseteq\{(h, l) \in$ $\left.\mathcal{E} \mid \exists i, j \in\{1, \ldots, m\}, i \neq j, h \in \mathcal{C}_{i}, l \in \mathcal{C}_{j}\right\}$. Precisely, the entries of $L$ and $P\left(t_{k}\right)$ satisfy the following relations:

$$
\begin{aligned}
& \left\{\begin{array}{l}
L_{i, j}=0, \text { if }(j, i) \notin \mathcal{E}_{L} \\
L_{i, j}<0, \text { if }(j, i) \in \mathcal{E}_{L}, i \neq j \\
L_{i, i}=-\sum_{j \neq i} L_{i, j}, \forall i \in\{1, \ldots, n\},
\end{array}\right. \\
& \left\{\begin{array}{l}
P_{i, j}\left(t_{k}\right)=0, \text { if }(j, i) \notin \mathcal{E}_{P}\left(t_{k}\right), i \neq j \\
P_{i, i}\left(t_{k}\right)>0, \forall i=\{1, \ldots, n\} \\
P_{i, j}\left(t_{k}\right)>0, \text { if }(j, i) \in \mathcal{E}_{P}\left(t_{k}\right), i \neq j \\
\sum_{j=1}^{n} P_{i, j}\left(t_{k}\right)=1, \forall i \in\{1, \ldots, n\} .
\end{array}\right.
\end{aligned}
$$

According to (3), given some $i$, if $P_{i, j}\left(t_{k}\right)=0$ for all $j \neq$ $i$ then $P_{i, i}\left(t_{k}\right)=1$, meaning that no jump occurs on the state of the agent $i$ at time $t_{k}$. The values $L_{i, j}$ and $P_{i, j}\left(t_{k}\right)$ represent the weight of the state of the agent $j$ in the updating process of the state of agent $i$ when using the continuous and discrete dynamics, respectively. The matrices $L$ and $P\left(t_{k}\right)$ describe the level of influence of each agent inside its cluster and outside it, respectively. So, $L$ is constant while $P$ varies in time depending on which agents udpate their state. The weight of influence $P_{i, j}$ may also vary in time for a same couple $(j, i)$.

It is worth noting that $L$ has the following block diagonal structure

$$
L=\left(\begin{array}{ccc}
L_{1} & & \\
& \ddots & \\
& & L_{m}
\end{array}\right), L_{i} \in \mathbb{R}^{n_{i}}
$$

with $L_{i} \mathbf{1}_{n_{i}}=\mathbf{0}_{n_{i}}$ and $P\left(t_{k}\right) \mathbf{1}_{m}=\mathbf{1}_{m}$.

\section{PRELIMINARIES}

In this section we introduce the assumptions required for the development presented in the next section and we recall the main result in [21] which is the basis of our stability analysis.

\section{A. Work assumptions}

In order to prove that the reset algorithm (1) guarantees asymptotic consensus for every initial condition $x_{0}$ we have to impose some standard assumptions. The first one concerns 
a minimal connectivity property of the whole network and of each cluster.

Assumption 1 (Network structure): The graph $\mathcal{G}=(\mathcal{V}, \mathcal{E})$ is such that

- For each cluster $C_{i}$, the induced graph $\left(C_{i}, \mathcal{E}_{L} \cap\left(C_{i} \times C_{i}\right)\right)$ contains a spanning tree with the root $r_{i}$,

- eventually re-ordering the clusters, the following holds: for all $i \geq 2$ there exist $j<i$ and $l_{i} \in \mathcal{C}_{j}$ such that $\left(l_{i}, r_{i}\right) \in \mathcal{E}$. We denote by

$$
\mathcal{E}_{T}=\left\{\left(l_{i}, r_{i}\right) \mid i \in\{2, \ldots, m\}\right\}
$$

the set of these $m-1$ such edges.

The previous assumption implies that $\mathcal{G}$ contains a spanning tree having the root in $\mathcal{C}_{1}$ (formed by the union of the spanning trees in each cluster together with the edges in $\left.\mathcal{E}_{T}\right)$. The assumption is satisfied if the induced graph of each cluster is strongly connected and so is $\mathcal{G}$. It also holds if the graph induced by the set of roots of all clusters contains a spanning tree.

The first part of Assumption 1 has a direct consequence on the continuous dynamics since equation (2) imposes $L_{i, j}<0$ when $(j, i) \in \mathcal{E}_{L}$. The second part of Assumption 1 guarantees the existence of the inter-cluster interaction structure formed by $\mathcal{E}_{T}$.

The next hypothesis of this work is standard in the literature (see [24]) and it ensures a minimal influence of the states implicated in the reset process of the agents.

Assumption 2 (Minimal influence): There exists a constant $\alpha \in(0,1)$ such that, for all reset times $t_{k}$, if $P_{i, j}\left(t_{k}\right) \neq$ 0 and $(i, j) \in \mathcal{E}_{T}$ then $P_{i, j}\left(t_{k}\right) \geq \alpha$.

Remark 1: As stated at the end of the previous section, $P_{i, j}$ can vary in time. Thus $P_{i, j},(i, j) \in \mathcal{E} \backslash \mathcal{E}_{L}$ can converge to zero. However, in order to reach consensus, the interaction between clusters should not completely vanish. In this context, Assumption 2 guarantees a minimal influence of one cluster on the root of some other cluster at the reset time.

In order to emphasize that agents belonging to the cluster $\mathcal{C}_{i}$ reset their state at time $t_{k}$, we use extraction function $\phi_{i}$ defined such that $t_{\phi_{i}(h)}, h \in \mathbb{N}$ is the $h$-th time an agent in cluster $\mathcal{C}_{i}$ resets its state, i.e.

$\phi_{i}(h)=\min \left\{k>\phi_{i}(h-1) \mid \exists j \in \mathcal{C}_{i}, \ell \in \mathcal{V} \backslash \mathcal{C}_{i}, P_{j, \ell}\left(t_{k}\right)>0\right\}$.

where for consistency, we imposed $\phi_{i}(-1)=-1$, for all $i \in\{1, \ldots, m\}$.

\section{B. Sufficient condition for consensus}

In order to guarantee consensus, two supplementary assumptions have been imposed in [21]. They simply state that inter-cluster influence is persistent in time and the root of one cluster does not present accumulations of reset instants (Zeno phenomenon). In the sequel, instead of imposing these assumptions we design event-triggering reset strategies that will satisfy them. In the following we recall the main result in [21] as well as the required assumptions.
Assumption 3: There exists a positive constant $\delta_{\max }>0$ satisfying the following: for all $(l, r) \in \mathcal{E}_{T}$,

- there exists $k \in \mathbb{N}$ such that $t_{k} \leq \delta_{\max }$ and $(l, r) \in$ $\mathcal{E}_{P}\left(t_{k}\right)$

- if $(l, r) \in \mathcal{E}_{P}\left(t_{k}\right)$ there exists $\tau \in\left[t_{k}, t_{k}+\delta_{\text {max }}\right]$ such that $(l, r) \in \mathcal{E}_{P}(\tau)$.

Assumption 4: There exists a positive constant $\delta<\delta_{\max }$ such that

$$
t_{\phi_{i}(k+1)}-t_{\phi_{i}(k)} \geq \delta, \forall i \in\{1, \ldots, m\} .
$$

In other words, there exists a lower bound for the period between the consecutive reset instants on the state of agents belonging to the same cluster. Notice that according to Assumptions 1 and 3 , all clusters in $\{2, \ldots, m\}$ reset an infinite number of times, so that for these clusters, $\phi_{i}$ is well defined. Cluster $\mathcal{C}_{1}$ may not reset an infinite number of times. In this case, $t_{\phi_{1}}(k)$ is only defined for $k$ smaller than some finite bound, and should still satisfy Assumption 4 for these $k$.

Let us introduce now the global diameter of the network at the time $t \in \mathbb{R}_{+}$:

$$
\Delta(t)=\max _{i \in \mathcal{V}} x_{i}(t)-\min _{i \in \mathcal{V}} x_{i}(t)
$$

Theorem 2 (Theorem 9 in [21]): Suppose that Assumptions $1,2,3$ and 4 hold. There exists some positive decay rate $\beta \in[0,1)$ such that for all $t \in \mathbb{R}_{+}$,

$$
\Delta\left(2(m+1) \delta_{\max }+t\right) \leq \beta \Delta(t)
$$

guaranteeing the consensus is asymptotically reached in the overall network.

\section{DESIGN OF EVENT TRIGGERED RESET RULE FOR CONSENSUS}

In order to avoid unnecessary inter-cluster communications we can define the reset sequence using an event-based strategy. In this section we propose two event based rules and we prove that they define reset sequences satisfying assumptions 3 and 4. This allows applying Theorem 2 to prove consensus subject to these reset rules.

The first rule defining the reset sequence is not fully decentralized. Each reseting element $r_{i}$ needs to know the states of all the agents in its own cluster $\mathcal{C}_{i}$ at the reset instants $t_{\phi_{i}(k)}$. This information is used to compute the local agreement value $x_{i}^{*}\left(t_{k}\right)$. It is worth noting that this information, which is centralized at cluster level, is needed only at isolated instants corresponding to resets. Indeed, the function $x_{i}^{*}(\cdot)$ is constant on the interval $\left[t_{\phi_{i}(k)}, t_{\phi_{i}(k+1)}\right)$. The second rule defining the reset sequence is fully decentralized since it requires only local/decentralized information. The reseting element $r_{i}$ of cluster $\mathcal{C}_{i}$ needs to know only the state of its neighbors. Nevertheless, each reseting element $r_{i}$ has to compute the maximal distance to its neighbors at every instant $t \in\left[t_{\phi_{i}(k)}, t_{\phi_{i}(k+1)}\right)$.

In the following we use the following instrumental assumption: 
Assumption 5: The components of the Laplacian matrix $L$ are uniformly bounded i.e. there exists $\bar{\alpha}>0$ finite real number such that $\left|L_{i, j}\right| \leq \bar{\alpha}, \forall i, j \in\{1, \ldots, n\}$.

This is necessary to ensure that during the continuous dynamics, the agents do not approach one to another indefinitely fast. We can notice that, in practice, this assumption is very natural and is almost always satisfied.

\section{A. Semi-decentralized event-triggered resets}

First, let us introduce the diameter of the cluster $\mathcal{C}_{i}$ as

$$
\Delta_{i}(t)=\max _{j \in \mathcal{C}_{i}} x_{j}(t)-\min _{j \in \mathcal{C}_{i}} x_{j}(t) .
$$

Definition 3: The distance between the node $r_{i}$ and the local agreement value of the cluster $\mathcal{C}_{i}$ at time $t$ is denoted by $d_{i}(t)=\left|x_{r_{i}}(t)-x_{i}^{*}(t)\right|$. Considering $\epsilon>0$ a fixed scalar, the reset sequence $\left(t_{k}\right)_{k \in \mathbb{N}}$ associated with the dynamics (1) is defined as follows: for all $i \in\{1, \ldots, m\}$ and for all $k \geq 0$,

- if $d_{i}\left(t_{\phi_{i}(k-1)}\right)>\epsilon$ we define

$$
t_{\phi_{i}(k)}=\min _{t \geq t_{\phi_{i}(k-1)}}\left\{d_{i}(t) \leq \frac{d_{i}\left(t_{\phi_{i}(k-1)}\right)}{a_{i}}\right\},
$$

- otherwise, $t_{\phi_{i}(k)}=t_{\phi_{i}(k-1)}+\delta$ with

$$
\delta=\min _{i \in\{1, \ldots, m\}} \frac{a_{i}-1}{a_{i}} \frac{\epsilon}{2 n_{i} \bar{\alpha} \Delta_{i}(0)},
$$

where the $a_{i}>1$ are design parameters fixed a priori. (We recall that for consistency, we denote $t_{\phi_{i}(-1)}=0$ ).

Remark 2: In our numerical illustrations we consider $a_{i}=$ $a_{j}, \forall i, j \in\{1, \ldots, m\}$ but these values can be used by the designer to change the reset frequency of some/all clusters.

Proposition 4: Let us consider the dynamics (1) under Assumptions 1, 2 and 5. Then, the associated reset sequence introduced by Definition 3 satisfies the Assumptions 3, 4 .

Proof: If $d_{i}\left(t_{\phi_{i}(k)}\right) \leq \epsilon$, the second point in Definition 3 applies and Assumptions 3, 4 hold. Otherwise, the first point applies and for a fixed cluster $\mathcal{C}_{i}, i \in\{1, \ldots, m\}$ we have to show that the reset sequence satisfies Assumptions $3,4$.

- Again, we start by proving that Assumption 4 holds. This means, a dwell time $\delta$ exists between a reset time $t_{\phi_{i}(k)}$ and the first time $t$ such that when $d_{i}(t) \leq \frac{d_{i}\left(t_{\phi_{i}(k)}\right)}{a_{i}}$.

We recall that for all $t \in\left[t_{\phi_{i}(k)}, t_{\phi_{i}(k+1)}\right)$ one has $\dot{x}_{i}^{*}(t)=$ 0 and

$$
\dot{x}_{r_{i}}(t)=-\sum_{j \in \mathcal{C}_{i}} L_{r_{i}, j}\left(x_{j}(t)-x_{r_{i}}(t)\right)
$$

Thus, using Assumption 5, one obtains that between two reset instants the following holds:

$$
\dot{d}_{i}(t) \geq-n_{i} \bar{\alpha} \Delta_{i}(t)
$$

Moreover, since $\Delta(\cdot)$ is non-increasing function one obtains that

$$
\dot{d}_{i}(t) \geq-n_{i} \bar{\alpha} \Delta_{i}(0) .
$$

Integrating the last equation we finally get that

$$
d_{i}(t) \geq d\left(t_{\phi_{i}(k)}\right)-n_{i} \bar{\alpha} \Delta_{i}(0)\left(t-t_{\phi_{i}(k)}\right) .
$$

Thus, in order to have $d_{i}(t) \leq \frac{d_{i}\left(t_{\phi_{i}(k)}\right)}{a_{i}}$ one needs

$$
\frac{d_{i}\left(t_{\phi_{i}(k)}\right)}{a_{i}} \geq d\left(t_{\phi_{i}(k)}\right)-n_{i} \bar{\alpha} \Delta_{i}(0)\left(t-t_{\phi_{i}(k)}\right)
$$

which is equivalent with

$$
\left(t-t_{\phi_{i}(k)}\right) \geq \frac{a_{i}-1}{a_{i}} \frac{d\left(t_{\phi_{i}(k)}\right)}{n_{i} \bar{\alpha} \Delta_{i}(0)}>\frac{a_{i}-1}{a_{i}} \frac{\epsilon}{n_{i} \bar{\alpha} \Delta_{i}(0)}
$$

This part of the proof finishes by choosing

$$
\delta=\min _{i \in\{1, \ldots, m\}} \frac{a_{i}-1}{a_{i}} \frac{\epsilon}{n_{i} \bar{\alpha} \Delta_{i}(0)} .
$$

- Let us show now that Assumption 3 also holds. Between $d_{i}\left(t_{\phi_{i}(k)}\right)$ and $d_{i}\left(t_{\phi_{i}(k+1)}\right)$ the overall dynamics of the cluster $\mathcal{C}_{i}$ is described by a fixed Laplacian matrix

$$
\dot{x}_{\mathcal{C}_{i}}(t)=-L_{i} x_{\mathcal{C}_{i}}(t)
$$

Thus, there exists $M_{i}>0$ and $\rho_{i}>0$ such that

$$
\Delta_{i}(t) \leq M_{i} e^{-\rho_{i}\left(t-t_{\phi_{i}(k-1)}\right)} \Delta_{i}\left(t_{\phi_{i}(k)}\right)
$$

where $\rho_{i}$ is the convergence speed (see for instance [25]) associated with the matrix $L_{i}$.

Moreover, one has $d_{i}(t) \leq \Delta_{i}(t), \forall i \in\{1, \ldots, m\}$ and $\forall t \geq 0$. Combining this with (5) yields

$$
d_{i}(t) \leq M_{i} e^{-\rho_{i}\left(t-t_{\phi_{i}(k-1)}\right)} \Delta_{i}\left(t_{\phi_{i}(k)}\right)
$$

Using (6), straightforward mathematical manipulation shows that $d_{i}(t)>\frac{d_{i}\left(t_{\phi_{i}(k)}\right)}{a_{i}}$ implies

$$
\begin{aligned}
t-t_{\phi_{i}(k-1)} & <\frac{1}{\rho_{i}} \ln \left(\frac{a_{i} M_{i} \Delta_{i}\left(t_{\phi_{i}(k)}\right)}{d_{i}\left(t_{\phi_{i}(k)}\right)}\right) \\
& <\frac{1}{\rho_{i}} \ln \left(\frac{a_{i} M_{i} \Delta_{i}(0}{\epsilon}\right) .
\end{aligned}
$$

The proof ends by defining

$$
\delta_{\max }=\max _{i \in\{1, \ldots, m\}} \frac{1}{\rho_{i}} \ln \left(\frac{a_{i} M_{i} \Delta_{i}(0}{\epsilon}\right) .
$$

\section{B. Fully decentralized event-triggered resets}

Definition 5: The distance between the node $r_{i}$ and its fairest neighbor in cluster $\mathcal{C}_{i}$ at time $t$ is denoted by

$$
\tilde{d}_{i}(t)=\max _{j \text { such that }\left(r_{i}, j\right) \in \mathcal{E}_{L}}\left|x_{r_{i}}(t)-x_{j}(t)\right| .
$$

Considering $\epsilon>0$ a fixed scalar, the reset sequence $\left(t_{k}\right)_{k \in \mathbb{N}}$ associated with the dynamics (1) is defined as follows: for all $i \in\{1, \ldots, m\}$ and for all $k \geq 0$,

- if $\tilde{d}_{i}\left(t_{\phi_{i}(k-1)}\right)>\epsilon$ we define

$$
t_{\phi_{i}(k)}=\min _{t \geq t_{\phi_{i}(k-1)}}\left\{\tilde{d}_{i}(t) \leq \frac{\tilde{d}_{i}\left(t_{\phi_{i}(k-1)}\right)}{a_{i}}\right\},
$$

- otherwise, $t_{\phi_{i}(k)}=t_{\phi_{i}(k-1)}+\delta$ with

$$
\delta=\min _{i \in\{1, \ldots, m\}} \frac{a_{i}-1}{a_{i}} \frac{\epsilon}{n_{i} \bar{\alpha} \Delta_{i}(0)},
$$


where the $a_{i}>1$ are design parameters fixed a priori. (We recall that for consistency, we denote $t_{\phi_{i}(-1)}=0$ ).

Remark 3: Once again we can fix $a_{i}=a_{j}, \forall i, j \in$ $\{1, \ldots, m\}$ or we can use $a_{i}$ to change the reset frequency of some/all clusters.

Proposition 6: Let us consider the dynamics (1) under Assumptions 1, 2 and 5. Then, the associated reset sequence introduced by Definition 5 satisfies the Assumptions 3, 4 .

Proof: The proof is similar to the one of Proposition 4. Nevertheless, some technical details have to be pointed out. If $\tilde{d}_{i}\left(t_{\phi_{i}(k)}\right) \leq \epsilon$, the second point in Definition 3 applies and Assumptions 3, 4 hold. Otherwise, the first point applies and for a fixed cluster $\mathcal{C}_{i}, i \in\{1, \ldots, m\}$ we have to show that the reset sequence satisfies Assumptions 3, 4.

- We start by proving that Assumption 4 holds. For any $j \in \mathcal{C}_{i}$ such that $\left(r_{i}, j\right) \in \mathcal{E}_{l}$ we define

$$
\tilde{d}_{i, j}\left(t_{\phi_{i}(k)}\right)=\left\|x_{r_{i}}\left(t_{\phi_{i}(k)}\right)-x_{j}\left(t_{\phi_{i}(k)}\right)\right\| .
$$

Let $\tilde{j} \in \mathcal{C}_{i}$ be one neighbor of $r_{i}$ such that $\tilde{d}_{i}\left(t_{\phi_{i}(k)}\right)=$ $\tilde{d}_{i, \tilde{j}}\left(t_{\phi_{i}(k)}\right)$. We emphasize that for all $t \in\left[t_{\phi_{i}(k)}, t_{\phi_{i}(k+1)}\right)$ one has

$$
\begin{aligned}
\dot{x}_{r_{i}}(t) & =-\sum_{j \in \mathcal{C}_{i}} L_{r_{i}, j}\left(x_{j}(t)-x_{r_{i}}(t)\right) \\
\dot{x}_{\tilde{j}}(t) & =-\sum_{j \in \mathcal{C}_{i}} L_{\tilde{j}, j}\left(x_{j}(t)-x_{\tilde{j}}(t)\right)
\end{aligned}
$$

yielding, as in the proof of Proposition 4,

$$
\dot{\tilde{d}}_{i, \tilde{j}}(t) \geq-2 n_{i} \bar{\alpha} \Delta_{i}(0) .
$$

Integrating this equation we get

$$
\tilde{d}_{i, \tilde{j}}(t) \geq \tilde{d}_{i, \tilde{j}}\left(t_{\phi_{i}(k)}\right)-2 n_{i} \bar{\alpha} \Delta_{i}(0)\left(t-t_{\phi_{i}(k)}\right) .
$$

But $\tilde{d}_{i}(t) \geq \tilde{d}_{i, \tilde{j}}(t), \forall t \geq 0$ and $\tilde{d}_{i}\left(t_{\phi_{i}(k)}\right)=\tilde{d}_{i, \tilde{j}}\left(t_{\phi_{i}(k)}\right)$ which leads as to

$$
\tilde{d}_{i}(t) \geq \tilde{d}_{i}\left(t_{\phi_{i}(k)}\right)-2 n_{i} \bar{\alpha} \Delta_{i}(0)\left(t-t_{\phi_{i}(k)}\right) .
$$

From this point we continue by mimicking the proof of Proposition 4. In order to have $\tilde{d}_{i}(t) \leq \frac{\tilde{d}_{i}\left(t_{\phi_{i}(k)}\right)}{a_{i}}$ one needs

$$
\frac{\tilde{d}_{i}\left(t_{\phi_{i}(k)}\right)}{a_{i}} \geq \tilde{d}\left(t_{\phi_{i}(k)}\right)-2 n_{i} \bar{\alpha} \Delta_{i}(0)\left(t-t_{\phi_{i}(k)}\right)
$$

which is equivalent with

$$
\left(t-t_{\phi_{i}(k)}\right) \geq \frac{a_{i}-1}{a_{i}} \frac{\tilde{d}\left(t_{\phi_{i}(k)}\right)}{2 n_{i} \bar{\alpha} \Delta_{i}(0)}>\frac{a_{i}-1}{a_{i}} \frac{\epsilon}{2 n_{i} \bar{\alpha} \Delta_{i}(0)}
$$

This part of the proof finishes by choosing

$$
\delta=\min _{i \in\{1, \ldots, m\}} \frac{a_{i}-1}{a_{i}} \frac{\epsilon}{2 n_{i} \bar{\alpha} \Delta_{i}(0)} .
$$

- To prove that Assumption 3 also holds, we simply notice that $\tilde{d}_{i}(t) \leq \Delta_{i}(t), \forall i \in\{1, \ldots, m\}$. Thus the arguments in the proof of Proposition 4 can be applied exactly in the same manner to define

$$
\delta_{\max }=\max _{i \in\{1, \ldots, m\}} \frac{1}{\rho_{i}} \ln \left(\frac{a_{i} M_{i} \Delta_{i}(0}{\epsilon}\right)
$$

that upper-bounds all the intervals $\left[t_{\phi_{i}(k)}, t_{\phi_{i}(k+1)}\right)$.

\section{ILLUSTRATIVE EXAMPLE}

In the following we consider a toy example that numerically validates the results reported in the paper. In this example the network contains 5 agents and it is partitioned in two clusters of 2 and 3 agents respectively. The directed graph capturing the interconnections during the continuous dynamics is described by the following generalized Laplacian matrix

$$
L=\left(\begin{array}{ccccc}
4 & -2 & -2 & 0 & 0 \\
-1 & 1 & 0 & 0 & 0 \\
0 & -2 & 2 & 0 & 0 \\
0 & 0 & 0 & 3 & -3 \\
0 & 0 & 0 & -1 & 1
\end{array}\right)
$$

which has a block diagonal structure corresponding to the two clusters. Each cluster contains only one node able to interact with agents outside its own cluster (node 1 in the first cluster and node 4 in the second cluster). The reset dynamics of the node 1 and 4 are:

$$
\begin{aligned}
& x_{1}\left(t_{\phi_{1}(k)}\right)=0.45 x_{1}\left(t_{\phi_{1}(k)}^{-}\right)+0.55 x_{5}\left(t_{\phi_{1}(k)}^{-}\right) \\
& x_{4}\left(t_{\phi_{2}(k)}\right)=0.25 x_{3}\left(t_{\phi_{2}(k)}^{-}\right)+0.75 x_{4}\left(t_{\phi_{2}(k)}^{-}\right) .
\end{aligned}
$$

Starting with the initial condition $x=[8,7,9,2,3]^{\top}$ and $a_{1}=a_{2}=2$, using the first reset rule we get the overall behavior of the network depicted in Figure 1. On this figure one can observe that reset instants are not synchronized and we may have several resets of the root of one cluster before the root of the second cluster jumps.

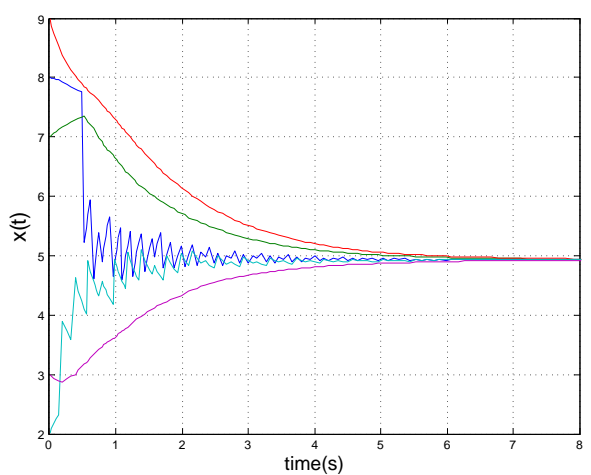

Fig. 1. Consensus of the five agents grouped in 2 clusters when the resets are defined by the semi-decentralized rule with $a_{1}=a_{2}=2$.

In order decrease the reset frequency and make even more clear the asynchrony of reset instants, we have changed the parameters occurring in the reset rule as follows $a_{1}=$ $200, a_{2}=2$. The behavior of the network is correspondingly modified as can be seen in Figure 2 .

Similar tests have been done for the same network with fully-decentralized reset instants and the results are presented in Figure 3.

It is worth noting that consensus value depends not only on the initial condition and interconnection topology but also on the reset sequence. 


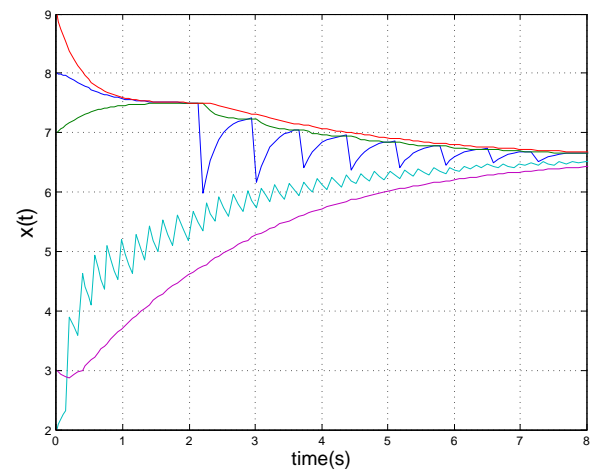

Fig. 2. Consensus of the five agents grouped in 2 clusters when the resets are defined by the semi-decentralized rule with $a_{1}=200, a_{2}=2$.
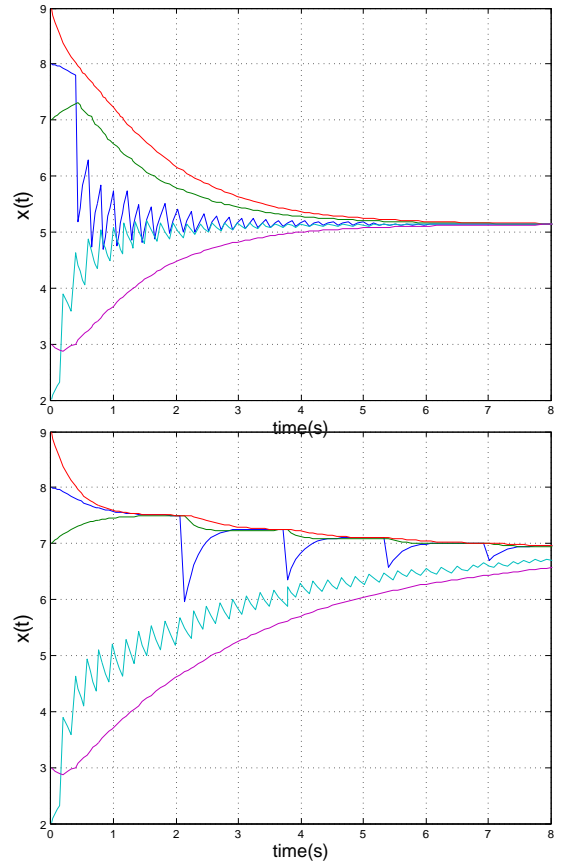

Fig. 3. Consensus of the five agents grouped in 2 clusters when the resets are defined by the fully-decentralized rule with Up: $a_{1}=a_{2}=2$. Down: $a_{1}=200, a_{2}=2$

\section{CONCLUSIONS}

In this paper we have analyzed the consensus in networks partitioned in several clusters. Each agent continuously interacts inside its cluster and some of them discretely reset their state by taking into account information from other clusters. Thus, we end up with heterogeneous networks of agents having linear dynamics and impulsive ones. We impose that the network satisfies some weak connectivity and bounded influence assumptions. In this framework the consensus is guaranteed as far as the reset sequence for each cluster is divergent and the period between two reset instants of the same cluster is lower-bounded by a strictly positive dwell-time. In order to define the sequences satisfying the aforementioned properties we use event-dependent and decentralized rules. A numerical example illustrates the results.

\section{REFERENCES}

[1] O. Ratmann, C. Wiuf, and J. W. Pinney, "From evidence to inference: Probing the evolution of protein interaction networks," HFSP Journal, vol. 3, no. 5, pp. 290-306, 2009

[2] G. Pavlopoulos, M. Secrier, C. Moschopoulos, T. Soldatos, S. Kossida, J. Aerts, R. Schneider, and P. G. Bagos, "Using graph theory to analyze biological networks," BioData Mining, vol. 4, no. 10, pp. 1-27, 2011.

[3] R. Hegselmann and U. Krause, "Opinion dynamics and bounded confidence models, analysis, and simulation," Journal of Artificial Societies and Social Simulation, vol. 5, no. 3, 2002.

[4] V. D. Blondel, J. M. Hendrickx, , and J. Tsitsiklis, "On Krause's multi-agent consensus model with state-dependent connectivity," IEEE Trans. on Automatic Control, vol. 54, no. 11, pp. 2586-2597, 2009.

[5] I.-C. Morărescu and A. Girard, "Opinion dynamics with decaying confidence: Application to community detection in graphs," IEEE Trans. on Automatic Control, vol. 56, no. 8, pp. 1862 - 1873, 2011.

[6] J.-M. Andreoli, "Coordination as negotiated transactions." Object Orientation with Parallelism and Persistence, pp. 151-173, 1995.

[7] H. Tanner, A. Jadbabaie, and G. Pappas, "Stable flocking of mobile agents part i: Dynamic topology," in Proceedings of the 42th IEEE Conference on Decision and Control, vol. 2, 2003, pp. 2010-2015.

[8] F. Bullo, J. Cortés, and S. Martinez, Distributed Control of Robotic Networks. A Mathematical Approach to Motion Coordination Algorithms. Princeton University Press, 2009.

[9] A. Jadbabaie, J. Lin, and A. S. Morse, "Coordination of groups of mobile autonomous agents using nearest neighbor rules," IEEE Trans. on Automatic Control, vol. 48, no. 6, pp. 988-1001, 2003.

[10] W. Ren and R. W. Beard, "Consensus seeking in multiagent systems under dynamically changing interaction topologies," IEEE Trans. on Automatic Control, vol. 50, no. 5, pp. 655-661, 2005.

[11] L. Moreau, "Stability of multiagent systems with time-dependent communication links," IEEE Trans. on Automatic Control, vol. 50, no. 2, pp. 169-182, 2005.

[12] D. Gfeller and P. D. L. Rios, "Spectral coarse graining and synchronization in oscillator networks," Phys. Rev. Lett., vol. 100, p. 174104 2008

[13] E. Steur, I. Tyukin, and H. Nijmeijer, "Semi-passivity and synchronizationof diffusively coupled neuronal oscillators," Physica D, no. 238, pp. 2119-2128, 2009.

[14] I.-C. Morărescu, W. Michiels, and M. Jungers, "Synchronization of coupled nonlinearoscillators with shifted gamma-distributed delays," in Proceedings of American Control Conference, 2013.

[15] H. Tanner, A. Jadbabaie, and G. Pappas, "Flocking in teams of nonholonomic agents," in Cooperative Control, ser. Lecture Notes in Control and Information Sciences, V. Kumar, N. Leonard, and A. Morse, Eds. Springer, 2005, vol. 309, pp. 458-460.

[16] L. Buşoniu and I.-C. Morărescu, "Consensus for black-box nonlinear agents using optimistic optimization," Automatica, vol. 50, no. 4, pp. 1201-1208, 2014

[17] M. Zavlanos and G. Pappas, "Distributed connectivity control of mobile networks," IEEE Transactions on Robotics, vol. 24, no. 6, pp. $1416-1428,2008$

[18] M. Fiacchini and I.-C. Morărescu, "Convex conditions on decentralized control for graph topology preservation," IEEE Transactions on Automatic Control, vol. 59, no. 6, pp. 1640-1645, DOI.10.1109/TAC.2013.22927192014.

[19] B. Touri and A. Nedic, "On approximations and ergodicity classes in random chains," IEEE Transactions on Automatic Control, vol. 57, no. 11, pp. 2718-2730, 2012.

[20] M. C. Bragagnolo, I.-C. Morărescu, J. Daafouz, and P. Riedinger, "Lmi sufficient conditions for the consensus of linear agents with nearlyperiodic resets," in Proc. of the American Control Conference, 2014

[21] I.-C. Morărescu, S. Martin, and A. Girard, "Coordination in networks of linear impulsive agents," in Proceedings of the 53rd Conference on Decision and Control, 2014.

[22] D. Dimarogonas, E. Frazzoli, and K. Johansson, "Distributed eventtriggered control for multi-agent systems," Automatic Control, IEEE Transactions on, vol. 57, no. 5, pp. 1291-1297, May 2012.

[23] G. Shi and K. Johansson, "Multi-agent robust consensus-part ii Application to distributed event-triggered coordination," in Decision and Control and European Control Conference (CDC-ECC), 2011 50th IEEE Conference on, Dec 2011, pp. 5738-5743.

[24] V. D. Blondel, J. M. Hendrickx, A. Olshevsky, and J. Tsitsiklis, "Convergence in multiagent coordination, consensus, and flocking," in Proc. IEEE Conf. on Decision and Control, 2005, pp. 2996-3000.

[25] A. Olshevsky and J. Tsitsiklis, "Convergence speed in distributed consensus and averaging," SIAM Journal of Control and Optimization, vol. 48, no. 1, pp. 33-55, 2009. 